\title{
EFECTO DE LA CERO LABRANZA SOBRE ALGUNAS ACTIVIDADES BIOLÓGICAS EN UN ALFISOL DEL SUR DE CHILE
}

\author{
Marysol Alvear Z. ${ }^{1}$, Mario Pino B. ${ }^{\text {, Carlos Castillo R. }}{ }^{\text {, Carmen Trasar-Cepeda }}{ }^{2}$, \\ Fernando Gil-Sotres ${ }^{3}$.
}
${ }^{1}$ Departamento de Ciencias Químicas. Universidad de La Frontera. Casilla 54-D
Temuco, Chile. Correo electrónico: alvear.marysol@gmail.com
${ }^{2}$ Laboratorios del Departamento de Bioquímica del Suelo, Instituto de Investigaciones Agrobiológicas de Galicia, CSIC, España.
${ }^{3}$ Departamento de Edafología y Química Agrícola. Universidad Santiago de Compostela. España.

\section{Effect of non-tillage on some biological activities in an Alfisol from Southern Chile}

Keywords: Non-tillage, soil enzyme activities, microbial biomass, N-mineralization.

\begin{abstract}
Due to the necessity to stabilize and protect the soil from degradation processes, the agronomic practices such non-tillage (NT) were applied. Microbial biomass (MB) and some enzyme activities were evaluated here because they are very sensitive to the changes taken place in the environment. The aim of this study was to measure some biological activities in an Alfisol of the Southern Chile under NT cropping. The biological activities evaluated were: carbon (BMC) and nitrogen (BMN) of microbial biomass, respectively), $\mathrm{N}$ - mineralization, dehydrogenase and catalase activities and some hydrolytic enzyme activities representative of different cycles of biological importance in soil (C, N, P, S). Soil organic $\mathrm{C}$ total $\mathrm{N}$ and $\mathrm{S}$ content also were evaluated. Soil samples were collected after harvest at three different depths (0-5, 5-10 and 10-20 cm), in soils cropped 4, 7 and 20 years under NT. The results showed an increment in organic C, N, S, N-mineralization and in the biological activities in the top soil $5 \mathrm{~cm}$ depth, with an increment of year under NT. In general, there was close relationships were found among almost all biological activities evaluated $(\mathrm{r}>0.8 ; \mathrm{p}<0.05)$.
\end{abstract}

Palabras claves: cero labranza, actividades enzimáticas, biomasa microbiana, mineralización de $\mathrm{N}$. 


\section{RESUMEN}

Dada la necesidad de estabilizar y proteger los suelos, de los procesos de degradación, han surgido prácticas agronómicas conservacionistas, entre ellas, la cero labranza (CL). La medición de la biomasa microbiana (BM), junto a la determinación de algunas actividades enzimáticas en el suelo, fueron evaluadas ya que, son muy sensibles a los cambios producidos en el entorno. El objetivo de este estudio fue determinar algunas actividades biológicas en un Alfisol del sur de Chile sometido a un manejo de CL. Las actividades evaluadas fueron carbono $(\mathrm{C})$ y nitrógeno $(\mathrm{N})$ de la biomasa microbiana (CBM y NBM, respectivamente), mineralización del $\mathrm{N}$, actividades dehidrogenasa y catalasa y algunas actividades enzimáticas hidrolíticas representativas de los diferentes ciclos de los elementos químicos de importancia biológica en suelos (C, N, P, S). Para ello se muestrearon suelos en postcosecha a diferentes profundidades $(0-5,5-10$ y $10-20 \mathrm{~cm})$, desde sitios cultivados con cuatro, siete y veinte años con manejo de CL. Los resultados mostraron un incremento en el C, N y azufre (S) orgánicos, en la mineralización de N y en las actividades enzimáticas evaluadas, en los primeros $5 \mathrm{~cm}$ de suelo, en la medida que aumentan los años de CL. Además, se encontró una alta correlación $(\mathrm{r}>0.8 ; \mathrm{p}<0.05)$ entre la mayoría de las actividades biológicas evaluadas.

\section{INTRODUCCIÓN}

Actualmente hay una preocupación mundial respecto a la acumulación de "gases efecto invernadero", lo que ha conducido a la consideración de políticas para disminuir las emisiones de éstos y su impacto sobre el cambio climático global. Uno de los principales gases efecto invernadero, el dióxido de carbono $\left(\mathrm{CO}_{2}\right)$, se ha incrementado significativamente. El mayor factor contribuyente ha sido la combustión de combustibles fósiles en la producción de energía, y una segunda fuente ha sido el uso del suelo (Sauerbeck, 2001). Por otra parte, ante la creciente demanda de alimentos, fibras y protección ambiental de una sociedad en constante expansión, y el empobrecimiento de los recursos naturales no renovables (Cerón y Melgarejo, 2005), generan la necesidad de realizar una agricultura sustentable (Acevedo y Silva, 2003).

La CL responde a la necesidad de mantener y/o mejorar la calidad de los recursos naturales en el proceso productivo agrícola (Acevedo y Silva, 2003). Sus ventajas han motivado a los productores a establecer esta práctica, para superar problemas físicos del suelo como erosión, compactación y retención de humedad. También para solucionar problemas derivados de la falta de materia orgánica (MO) y fertilidad, ya que al mantener los rastrojos sobre el suelo, se evita la erosión y se estimula la actividad biológica aumentando la productividad del suelo (Crovetto, 1996; Crovetto, 2002). La fertilidad de un suelo también está dada por la cantidad y calidad de microorganismos y enzimas presentes o lo que es llamado fertilidad biológica de un suelo (Borie, 1994). Por lo tanto, para estimar el 'grado' de calidad se suele recomendar evaluar la actividad biológica del suelo (Trasar-Cepeda et al., 1998; Alvear et al., 2005; Joergensen y Emmerling, 2006).

Se sabe que bajo condiciones medio-ambientales apropiadas, la intensidad de reciclaje 'turnover time (TT)' de los productos orgánicos está controlada por el tamaño y actividad de la BM (Martens, 1995). Dada la importancia del TT para el flujo de $\mathrm{C}$ en el suelo, es importante su determinación. Gran número de reacciones bioquímicas, tienen un rol importante en el 
ciclado y disponibilidad de nutrientes que la planta utiliza, así como las que están involucradas en la transformación de la MO, las que son catalizadas por enzimas. Se sabe que algunas enzimas del suelo proceden tanto de animales como de las raíces de plantas y microorganismos, se acepta que la mayor parte deriva esencialmente de los microorganismos (Tabatabai, 1994). Se han encontrado muy buenas correlaciones entre la actividad microbiana del suelo y los niveles de actividad enzimática (Salam et al., 1999, Kandeler et al., 1999, Alvear et al., 2005). Entre los muchos factores que pueden afectar a las actividades enzimáticas del suelo tienen especial importancia la especie cultivada y el uso de enmiendas (AcostaMartínez y Tabatabai, 2000). Diversos estudios sobre la actividad de las enzimas extracelulares en diversos ecosistemas han mostrado que los productos químicos utilizados en agricultura (fertilizantes, pesticidas, herbicidas, etc.) tienen una influencia muy marcada sobre las enzimas del suelo (Tabatabai, 1994; Alvear et al., 2006).

La degradación de los sustratos orgánicos introducidos en el suelo consiste en una serie de reacciones oxidativas. Mediante la acción de dehidrogenasas y de los sistemas citocromos, los electrones son transferidos a través de una cadena de portadores intermedios hasta el oxígeno, que en medios aerobios actúa como aceptor final. Así, la dehidrogenasa, que es una actividad localizada intracelularmente, se considera como un índice de la actividad microbiana total del suelo (García-Izquierdo et al., 2003).

Por su parte, la catalasa se encuentra en todas las bacterias aeróbicas y en la mayor parte de los organismos anaerobios facultativos; es una enzima de tipo intracelular, de ahí que, se ha utilizado como índice de la biomasa edáfica y como un componente de diferentes índices de fertilidad (García-Izquierdo et al., 2003), al igual que la dehidrogenasa.

Las fosfomonoesterasas ácidas, hidrolizan el P orgánico que habitualmente se encuentra en el suelo a formas inorgánicas que pueden ser absorbidas por las plantas. En el suelo, éstas se consideran que son extracelulares o abióticas (Skujins, 1978), cuya actividad se ve influida por el manejo del suelo (García-Izquierdo et al., 2003) y presentan una tendencia a disminuir con la intensidad de labores agrícolas como la aradura (Doran, 1980).

Por otra parte, la fosfodiesterasa cataliza la reacción de hidrólisis de los di-ésterfosfatos a mono-éster-fosfatos; con gran aptitud para la degradación de ácidos nucleicos, por lo que su papel en el ciclo del P es importante (García-Izquierdo et al., 2003).

La arilsulfatasa cataliza la ruptura del enlace S-O. El rol de ésta en el ciclo del S edáfico, es importante, dado que más del $50 \%$ del S presente en los suelos aparece bajo la forma de éster-sulfato, es decir, bajo formas que pueden ser convertidas en sulfatos inorgánicos mediante la acción de álcalis en caliente (Freney, 1961).

La determinación de la carboximetilcelulasa (CM-celulasa) en suelos, se fundamenta en la valoración de azúcares reductores generados a partir de la hidrólisis de enlaces glucosídicos del interior de las moléculas cristalinas de celulosa (GarcíaIzquierdo et al., 2003).

La $\beta$-glucosidasa cataliza la hidrólisis de $\beta$-D-glucósidos, dando como producto glucosa, que es una fuente importante de energía para los microorganismos del suelo (Tabatabai, 1982).

La invertasa, hidroliza disacáridos de tipo $\beta$-furanósidos y se la ha relacionado con el número de microorganismos en el suelo y con las actividades metabólicas (Skujins, 1978), aunque se reconoce que ésta se encuentra fuertemente adsorbida por las partículas del suelo.

La ureasa cataliza la hidrólisis de la urea a $\mathrm{CO}_{2}$ y amonio. Se encuentra, en microorganismos, células animales y vege- 
tales. El interés por ésta se debe a su relación con el ciclo del $\mathrm{N}$ y por el amplio uso que se ha dado a la urea como fertilizante nitrogenado en la agricultura (GarcíaIzquierdo et al., 2003).

Por otra parte, la actividad proteasa del suelo es la responsable de la descomposición progresiva del $\mathrm{N}$ contenido en las proteínas; procede de diferentes tipos de microorganismos, de residuos de las plantas y de la mesofauna del suelo. Las proteasas se clasifican, de acuerdo con el sustrato sobre el que actúan, sean éstos simples como caseína o más complejos como la N-benzoil-L-arginina amida (BAA), que parece estar relacionada con la actividad proteolítica ligada a los coloides húmicos.

La labranza convencional con rompimiento del suelo produce una aceleración en los procesos de descomposición del C orgánico, por oxidación prematura de la MO (Crovetto, 1996). Dada la necesidad de proteger los suelos, han surgido en Chile prácticas agronómicas conservacionistas, entre ellas la CL.

El objetivo de este estudio consistió en determinar CBM y NBM, mineralización del $\mathrm{N}$, actividades dehidrogenasa y catalasa, junto con algunas actividades enzimáticas hidrolíticas, en un Alfisol del sur de Chile sometido a un manejo de cuatro, siete y veinte años de CL.

\section{MATERIALES Y METODOS}

Después de la cosecha del triticale (4 años de CL), del lupino (7 años de CL) y del trigo (20 años de CL), a fines de junio de 1999, se muestreó un Alfisol de la localidad de Florida, VIII Región, Chile (36 49'S, $72^{\circ} 40^{\prime} \mathrm{O}, 280 \mathrm{msnm}$ ), en parcelas sometidas a CL con mantención de rastrojos, durante cuatro, siete y veinte años. El suelo de cuatro años presentaba una rotación triticale-lupino-triticale; el de siete años, tres años de trigo y un año de lupino y el de veinte años, maíz y trigo, bajo riego. Las mues- tras de suelo de cada parcela se obtuvieron a partir de 6 submuestras, con la ayuda de un barreno de $3,5 \mathrm{~cm}$ de diámetro evaluándose a tres profundidades: $0-5 \mathrm{~cm} ; 5-10 \mathrm{~cm}$ y $10-20 \mathrm{~cm}$, guardadas en bolsas isotérmicas y refrigeradas hasta su análisis. Se determinó $\mathrm{C}, \mathrm{N}$ y $\mathrm{S}$ orgánicos, mediante analizador elemental, por combustión seca. La determinación del CBM se llevó a cabo mediante fumigación-extracción con $\mathrm{CHCl}_{3}$ con posterior medición del $\mathrm{C}$ total por oxidación de dicromato (Vance et al., 1987), utilizando como valor de $\mathrm{K}_{\mathrm{ec}} 0,45$ (Joergensen, 1996; Alvear et al., 2005) y el NBM se llevo a cabo utilizando el procedimiento descrito por Badalucco et al. (1992), utilizando como valor $\mathrm{K}_{\mathrm{en}}$ 0,54 (Brookes et al., 1985).

Para determinar la mineralización del $\mathrm{N}$, muestras de $10 \mathrm{~g}$ de suelo en duplicado fueron extraídas por $30 \mathrm{~min}$ con $50 \mathrm{ml}$ de $\mathrm{KCl} 2 \mathrm{M}$ antes y después de la incubación por 10 días a $25^{\circ} \mathrm{C}$, posteriormente el $\mathrm{N}$ amoniacal y el $\mathrm{N}$ inorgánico total fueron determinados en los extractos obtenidos por destilación de Kjeldahl. La mineralización fue determinada de la diferencia entre los valores obtenidos antes y después de la incubación de acuerdo al método descrito por Keeney y Bremner (1966).

Las diferentes actividades enzimáticas estudiadas, se midieron espectrofotométricamente, excepto la ureasa y la proteasa-BAA, actividades en que se utilizó un electrodo selectivo para cuantificar amoníaco. La actividad dehidrogenasa se determinó midiendo el trifenil formazán formado de acuerdo al método descrito por García-Izquierdo et al. (2003). La determinación de la actividad catalasa, se basa en su aptitud para descomponer el agua oxigenada en agua y oxígeno. La 4-aminoantipirina es oxidada, reaccionando con el fenol para dar un compuesto de color rosa que se puede medir a $505 \mathrm{~nm}$ (TrasarCepeda et al., 1999). La fosfomonoesterasa ácida y la fosfodiesterasa fueron determi- 
nadas por los métodos descritos por Alvear et al. (2005) y por Bowman y Tabatabai (1978), respectivamente. En cuanto a la arilsulfatasa, por el método de Tabatabai y Bremner (1970). La $\beta$-glucosidasa fue determinada de acuerdo al método descrito por Alvear et al.(2005), y la invertasa junto con la CM-celulasa fueron determinadas por el método de Schinner y von Mersi (1990). La ureasa fue determinada de acuerdo al método descrito por Gil-Sotres et al. (1992), y la proteasa-caseína junto con la proteasa que hidroliza $\mathrm{N}$ - $\alpha$-benzoíl-L-arginin-amida (BAA) determinadas por el método descrito por Ladd y Buttler (1972).

Dado el alto número de actividades biológicas descritas, para facilitar el análisis de los resultados y discusión, de acuerdo con la clasificación realizada por Nannipieri et al. (1995) quienes las agruparon en actividades generales de la actividad microbiana del suelo, grupo en el que se incluyen CBM, NBM, mineralización del $\mathrm{N}$, actividades enzimáticas oxido-reductasas y el grupo correspondiente a las actividades específicas, grupo que incluye a una gran cantidad de enzimas hidrolíticas.

Todas las determinaciones se realizaron en triplicado para cada submuestra y se expresaron en base a suelo seco $\left(105^{\circ} \mathrm{C}\right.$ por $24 \mathrm{~h}$ ). Los datos se normalizaron antes del análisis estadístico mediante la transformación arcoseno. Para todas las variables, posterior al análisis de varianza de dos factores, se aplicó el test de Duncan de rango múltiple $(\mathrm{p}<0,05)$. Para realizar los cálculos para el análisis estadístico se utilizó la versión de prueba del software estadístico SPSS 11.0 para Windows.

\section{RESULTADOS Y DISCUSIÓN}

La muestra de suelo con 20 años de CL presentaron los mayores valores de $\mathrm{C}, \mathrm{N}$ y $\mathrm{S}$ orgánicos en los primeros $5 \mathrm{~cm}$ de profundidad (Cuadro 1). Esta cantidad de C orgánico en el tratamiento 20 años CL con resi- duos podría ser atribuido a una menor tasa de descomposición de la MO como consecuencia de una distribución diferente de la MO en el perfil de suelo (Vidal et al., 1997, Borie et al., 2000). Acevedo y Silva (2003), indican que la CL contribuye a aumentar la captura $\mathrm{C}$ en el suelo, disminuyendo así las emisiones de $\mathrm{CO}_{2}$ a la atmósfera. $\mathrm{Al}$ analizar suelos sometidos durante varios años a CL, Roldán et al. (2003), encontraron niveles de $\mathrm{C}$ y $\mathrm{N}$ orgánicos significativamente mayores a los encontrados en un suelo sometido a labranza tradicional. Por su parte, Alvear et al. (2005) encontraron que el S orgánico, también es mayor en suelos sometidos a CL. Además, a medida que aumenta la profundidad del suelo, los valores obtenidos tanto para el C, N y S orgánicos son menores (Cuadro 1). En las muestras con 7 años de CL, la rotación incluyó varios años de cultivos con cereales, cuyos rastrojos contienen más lignina que los provenientes de leguminosas. Considerando que la lignina es altamente resistente a la descomposición microbiana (Melillo et al., 1982), y que el aumento de la concentración de lignina reduce la descomposición y la liberación de nutrientes desde los rastrojos (Vidal y Troncoso, 2003); lo que explicaría los menores contenidos de $\mathrm{C}, \mathrm{N}$ y $\mathrm{S}$ orgánicos a los obtenidos en las muestras con 4 años de CL. Esta última rotación también incluyó cereales, pero en una período más corto. Nuestros resultados fueron en línea con lo obtenido por Zagal et al. (2002), al comparar el \% de C orgánico entre dos rotaciones con diferente cantidad de cereales y leguminosas. Un cereal secuestra más C del suelo que una leguminosa, por lo que el 'input' promedio por año es menor en una rotación con mayor proporción de cereales. La baja relación $\mathrm{C}: \mathrm{N}$ de los residuos de leguminosas (7 años de CL), fomentan la actividad microbiana de las bacterias (Mera y Rouanet, 2003), éstas al no consumir $\mathrm{C}$, aumenta el secuestro de este nutriente en el suelo. 
Cuadro 1: Contenido de $\mathrm{C}, \mathrm{N}$ y $\mathrm{S}$ y relación $\mathrm{C} / \mathrm{N}$ a diferentes profundidades de un Alfisol sometido a un manejo bajo cuatro, siete y veinte años de CL.

Table 1: $\mathrm{C}, \mathrm{N}$ and $\mathrm{S}$ contents and $\mathrm{C} / \mathrm{N}$ ratio at different dephts in an Alfisol under four, seven and twenty years with NT.

\begin{tabular}{|c|c|c|c|c|c|}
\hline Suelo & $\begin{array}{c}\text { Profundidad } \\
(\mathrm{cm})\end{array}$ & $\begin{array}{c}\mathrm{C} \\
(\%)\end{array}$ & $\begin{array}{c}\mathrm{N} \\
(\%)\end{array}$ & $\begin{array}{c}\mathrm{S} \\
(\%)\end{array}$ & $\mathrm{C}: \mathrm{N}$ \\
\hline 4 años CL & $0-5$ & $3,75 \mathrm{~b}$ & $0,31 \mathrm{~b}$ & $0,098 \mathrm{~b}$ & 12,10 \\
\hline 7 años CL & $0-5$ & $2,84 \mathrm{c}$ & $0,23 \mathrm{c}$ & $0,071 \mathrm{c}$ & 12,35 \\
\hline 20 años CL & $0-5$ & $7,42 \mathrm{a}$ & $0,65 \mathrm{a}$ & $0,150 \mathrm{a}$ & 11,42 \\
\hline 4 años CL & $5-10$ & $2,64 \mathrm{~b}$ & $0,23 \mathrm{~b}$ & $0,042 \mathrm{~b}$ & 11,48 \\
\hline 7 años CL & $5-10$ & $1,86 \mathrm{c}$ & $0,16 \mathrm{c}$ & $0,033 \mathrm{c}$ & 11,63 \\
\hline 20 años CL & $5-10$ & $4,82 \mathrm{a}$ & $0,45 \mathrm{a}$ & $0,067 \mathrm{a}$ & 10,71 \\
\hline 4 años CL & $10-20$ & $2,34 \mathrm{a}$ & $0,21 \mathrm{a}$ & $0,041 \mathrm{ab}$ & 11,14 \\
\hline 7 años CL & $10-20$ & $1,41 \mathrm{bc}$ & $0,13 \mathrm{~b}$ & $0,025 \mathrm{c}$ & 10,85 \\
\hline 20 años CL & $10-20$ & $1,80 \mathrm{~b}$ & $0,18 \mathrm{~b}$ & $0,048 \mathrm{a}$ & 10,00 \\
\hline
\end{tabular}

En cada columna, para cada profundidad, medias con distinta letra indincan diferencia significativa (Test de Duncan de rango múltiple, <0,05).

Los valores de CBM más altos se obtuvieron en los primeros $5 \mathrm{~cm}$ de profundidad, en el suelo sometido a CL por 4 años (Cuadro 2). En los 5 a $10 \mathrm{~cm}$ de profundidad el CBM aumento en la medida que aumentaban los años de CL, mientras que de 10 a $20 \mathrm{~cm}$ de profundidad, disminuyó, con mayor número de años manejado con CL, lo que podría explicarse por la formación de capas superiores de suelo. Según Vidal et al. (1997), se produce un aumento significativo del CBM en el sistema CL comparado con la labranza tradicional, resultados que pueden explicarse por la presencia de residuos del cultivo anterior (lupino), ya que los manejos que favorecen la acumulación de MO en el suelo incrementan la cantidad de BM (Jenkinson y Ladd, 1981). Resultados similares fueron obtenidos por Saffigna et al. (1989) y Vidal et al. (1997), quienes consideran que el aumento de CBM en un sistema CL sin quema de residuos se debe a que éstos, mejoran las condiciones físicas del suelo, lo que genera una mayor retención de humedad y por ende se dispone de una mayor cantidad de sustratos carbonados.

Hubo un incremento del NBM (Cuadro 2) a mayor cantidad de años bajo CL. Por otra parte, a medida que la profundidad aumentaba, los valores obtenidos para el NBM fueron menores, resultados que concuerdan con los obtenidos por Vidal et al.(1997), quienes han analizado los efectos que las distintas técnicas agronómicas tienen sobre el CBM y NBM al evaluar el tipo de labranza (convencional y CL), manejo de residuos (retención y remoción) y sistemas de rotación. Además, encontraron que el sistema 
CL incrementó en un 30 a $40 \%$ la BM y la retención de residuos en un 20 a $30 \%$, destacándose el sistema de CL con retención de residuos como el tratamiento con mayores valores de CBM y NBM, en comparación con suelo sometido a labranza tradicional. Los valores obtenidos en este estudio, son superiores a los encontrados por Sparling et al. (1994) y Joergensen (1996).
Además, el NBM de este Alfisol se encuentra por sobre los valores reportados por Ojeda (1996) para suelos de la IX Región, manejados con CL y bosque nativo. Por su parte, el NBM fue significativamente más alto en los tratamientos con 7 y 20 años de CL, resultados similares a los informado por Smith y Paul (1990), Borie (1994) y Vidal et al.(1997).

Cuadro 2: Determinación de CBM y NBM, a diferentes profundidades de un Alfisol sometido a un manejo de cuatro, siete y veinte años bajo cero labranza.

Table 2: Microbial biomass $\mathrm{C}$ and MBN measurements at different dephts in an Alfisol under four, seven and twenty years with NT.

\begin{tabular}{|c|c|c|c|}
\hline SUELO & Profundidad $(\mathrm{cm})$ & $\begin{array}{c}\text { CBM } \\
\left(\mathrm{mg} \mathrm{C} \mathrm{kg}^{-1}\right)\end{array}$ & $\begin{array}{c}\text { NBM } \\
\left(\mathrm{mg} \mathrm{N} \mathrm{kg}^{-1}\right)\end{array}$ \\
\hline 4 años & $0-5$ & $500,9 \mathrm{a}$ & $90,9 \mathrm{c}$ \\
\hline 7 años & $0-5$ & $281,1 \mathrm{c}$ & $189,3 \mathrm{~b}$ \\
\hline 20 años & $0-5$ & $467,7 \mathrm{ab}$ & $265,3 \mathrm{a}$ \\
\hline 4 años & $5-10$ & $160,9 \mathrm{c}$ & $19,6 \mathrm{c}$ \\
\hline 7 años & $5-10$ & $301,3 \mathrm{~b}$ & $110,9 \mathrm{~b}$ \\
\hline 20 años & $5-10$ & $393,1 \mathrm{a}$ & $221,1 \mathrm{a}$ \\
\hline 4 años & $10-20$ & $158,4 \mathrm{a}$ & $43,5 \mathrm{c}$ \\
\hline 7 años & $10-20$ & $124,0 \mathrm{~b}$ & $62,6 \mathrm{a}$ \\
\hline 20 años & $10-20$ & $107,0 \mathrm{c}$ & $51,4 \mathrm{~b}$ \\
\hline
\end{tabular}

CBM y NBM: Carbono y nitrógeno en la biomasa microbiana, respectivamente. En cada columna, para cada profundidad, medias con distinta letra indincan diferencia significativa (Test de Duncan de rango múltiple, <0,05). 
Respecto a la mineralización del $\mathrm{N}$ orgánico, inicialmente el contenido de $\mathrm{N}-\mathrm{NH}_{4}+$ de los suelos es mayor a medida que aumenta el número de años de CL (Cuadro 3), lo mismo que, para el contenido de $\mathrm{N}$ final, hay diferencias significativas $(\mathrm{p}<0,05)$. Las cantidades de $\mathrm{N}$ mineralizado, que aparecen bajo formas amoniacales en los primeros $5 \mathrm{~cm}$ de suelo, aumentaron en la medida que aumentaban los años de
CL y el N inorgánico se comporto de la misma manera, valores dentro del rango reportado por otros investigadores para suelos no intervenidos (Groffman et al., 1996), lo que indica que, la CL es un sistema de producción que no afecta negativamente las características naturales de los suelos, a diferencia del efecto que tiene la labranza tradicional en las propiedades del suelo, luego de ser intervenido.

Cuadro 3: Mineralización del $\mathrm{N}$ a diferentes profundidades de un Alfisol sometido a un manejo de cuatro, siete y veinte años bajo CL. Resultados expresados en $\mathrm{mg} \mathrm{kg} .{ }^{1}$ de suelo.

Table 3: Nitrogen mineralization at different dephts in an Alfisol under four, seven and twenty years with NT.

\begin{tabular}{|c|c|c|c|c|c|c|c|}
\hline \multirow[t]{2}{*}{ Tratamiento } & \multirow{2}{*}{$\begin{array}{c}\text { Profundidad } \\
\mathrm{cm}\end{array}$} & \multicolumn{2}{|c|}{$\mathrm{N}$ inicial } & \multicolumn{2}{|c|}{$\mathrm{N}$ final } & \multicolumn{2}{|c|}{$\mathrm{N}$ mineralizado } \\
\hline & & $\mathrm{N}-\mathrm{NH}_{4}^{+}$ & $\mathrm{N}$-inorg & $\mathrm{N}-\mathrm{NH}_{4}{ }^{+}$ & $\mathrm{N}$-inorg & $\mathrm{N}-\mathrm{NH}_{4}^{+}$ & $\mathrm{N}$-inorg \\
\hline 4 años & $0-5$ & $1,33 \mathrm{c}$ & $45,23 \mathrm{a}$ & $3,97 \mathrm{~b}$ & $63,59 \mathrm{a}$ & $2,64 \mathrm{c}$ & $18,36 \mathrm{bc}$ \\
\hline 7 años & $0-5$ & $2,06 \mathrm{a}$ & $32,98 \mathrm{~b}$ & $5,36 \mathrm{a}$ & $53,60 \mathrm{~b}$ & $3,30 \mathrm{~b}$ & $20,62 \mathrm{~b}$ \\
\hline 20años & $0-5$ & $1,82 \mathrm{~b}$ & $30,49 \mathrm{~b}$ & $5,89 \mathrm{a}$ & $63,90 \mathrm{a}$ & $4,07 \mathrm{a}$ & $33,41 \mathrm{a}$ \\
\hline 4 años & $5-10$ & $1,28 \mathrm{~b}$ & $37,08 \mathrm{a}$ & $5,08 \mathrm{a}$ & $48,69 \mathrm{a}$ & $3,80 \mathrm{a}$ & $11,61 \mathrm{~b}$ \\
\hline 7 años & $5-10$ & $0,79 \mathrm{c}$ & $13,18 \mathrm{c}$ & $3,92 \mathrm{c}$ & $21,54 \mathrm{c}$ & $3,13 \mathrm{ab}$ & $7,73 \mathrm{c}$ \\
\hline 20 años & $5-10$ & $1,68 \mathrm{a}$ & $24,34 b$ & $4,18 b$ & $43,48 \mathrm{~b}$ & $2,50 \mathrm{c}$ & $19,14 \mathrm{a}$ \\
\hline 4 años & $10-20$ & $0,80 \mathrm{~b}$ & $25,06 \mathrm{a}$ & $3,56 \mathrm{a}$ & $26,49 \mathrm{a}$ & $2,76 \mathrm{a}$ & $1,43 \mathrm{c}$ \\
\hline 7 años & $10-20$ & $0,79 \mathrm{~b}$ & $11,06 \mathrm{~b}$ & $1,97 \mathrm{c}$ & $15,80 \mathrm{~b}$ & $0,64 \mathrm{c}$ & $4,74 \mathrm{~b}$ \\
\hline 20 años & $10-20$ & $1,14 \mathrm{a}$ & $7,20 \mathrm{c}$ & $2,70 \mathrm{~b}$ & $12,36 \mathrm{c}$ & $1,56 \mathrm{~b}$ & $5,16 a$ \\
\hline
\end{tabular}

N-inorg: N inorgánico.

En cada columna, para cada profundidad, medias con distinta letra indican diferencia significativa (Test de Duncan de rango múltiple, $\mathrm{p}<0,05$ ). 
En relación a las actividades enzimáticas evaluadas, se puede apreciar que el suelo bajo CL, a mayor cantidad de años, muestra una mayor actividad enzimática, principalmente en los primeros $5 \mathrm{~cm}$ de suelo, con una disminución importante al aumentar la profundidad (Figuras 1, 2, 3, 4 y 5). Lo anterior, era previsible ya que las actividades biológicas del suelo dependen de las fuentes de C lábil, por lo que se espera que su distribución sea paralela a la distribución de éste en el suelo (Franzleubbers, 2002).

Los valores más altos obtenidos para la actividad dehidrogenasa fueron para 20 años CL en los primeros $5 \mathrm{~cm}$ de suelo (Figura $1)$, valores similares a los reportados por Alvear et al. (2005), para la misma época de muestreo en un Ultisol de la IX Región sometido a tres años de CL. Por su parte, Ojeda (1996), informó valores de 264,0 y $504,0 \mu \mathrm{g}$ de rojo formazán $\mathrm{g}^{-1}$, para un suelo con trigo bajo CL y bosque nativo, respectivamente.

La actividad catalasa (Figura 1) en los primeros $5 \mathrm{~cm}$ fue de 2,31 a 2,99 $\mu$ moles $\mathrm{H}_{2} \mathrm{O}_{2} \mathrm{~g}^{-1} \mathrm{~h}^{-1}$. No existen datos de ésta actividad enzimática en suelos chilenos, sin embargo, al compararlos con suelos gallegos, nuestros valores son inferiores (TrasarCepeda et al., 1999). Por su parte, es importante determinar esta actividad enzimática pues, Gawronska et al. (1992) indican que, esta enzima es sensible, con fuertes reducciones, a las prácticas agrícolas como el monocultivo sostenido por largos períodos de tiempo.

La actividad fosfomonoesterasa ácida (Figura 2), presentó valores menores a los reportados para este mismo suelo en el año 1992 (Crovetto, 1992), en un suelo con 7 años de CL. En general, se observa que a mayor número de años bajo CL, mayor actividad fosfomonoesterasa ácida, siendo más altos los valores en los primeros $5 \mathrm{~cm}$ de suelo, debido al efecto de la cobertura que queda sobre éste luego de la cosecha (Crovetto, 2002). El comportamiento fren-

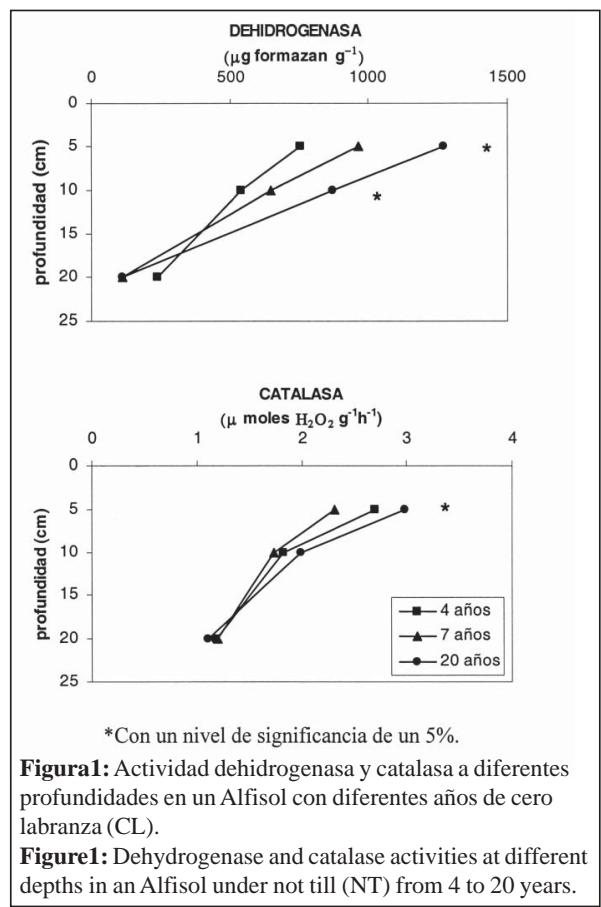

te a la adición de residuos orgánicos, ha sido discutido por diversos autores; para algunos, la actividad fosfomonoesterasa ácida se incrementa con la adición al suelo de productos orgánicos residuales (Perucci et al., 1984), lo que concuerda con lo obtenidos en este estudio. En cambio, Olander y Vitousek (2000), indican que esta actividad enzimática disminuye si en el suelo hay una mayor disponibilidad de $\mathrm{P}$ (retro-inhibición negativa). En cuanto a la actividad fosfodiesterasa es primera vez determinada en suelos chilenos (Figura 2), siendo menores a los reportados por Trasar-Cepeda et al. (2000).

La actividad arilsulfatasa (Figura 3), presenta valores similares a los encontrados por Pulford y Tabatabai (1988) y GarcíaAlvarez e Ibáñez (1994). En las muestras con 20 años de CL, en los primeros $5 \mathrm{~cm}$ de suelo, se obtuvieron valores superiores a los encontrados por Alvear et al. (2005), a diferencia de lo encontrado para las muestras con 4 y 7 años de CL, cuyos valores fueron similares. Acosta-Martínez et al. (2006) se- 


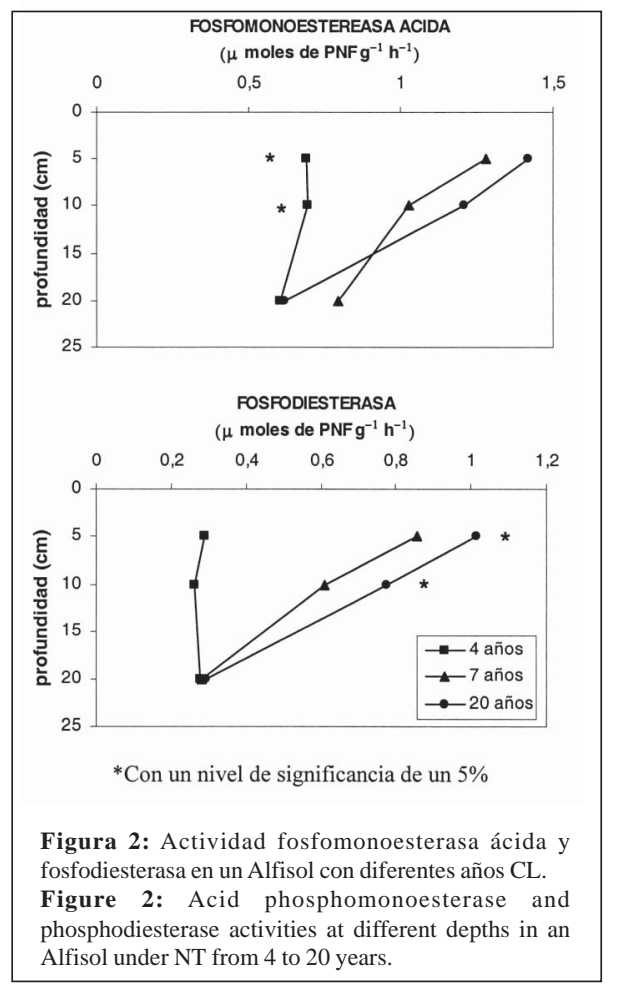

ñalan que la actividad arilsulfatasa es afectada por el tipo de uso al que sean sometidos los suelos de origen volcánico (forestal, pradera o agrícola). En forma similar a otras actividades enzimáticas, se ha observado que la arilsulfatasa aparece relacionada con el contenido de $\mathrm{C}$, la cual disminuye en profundidad en el suelo (Dick et al., 1988).

La actividad $\beta$-glucosidasa (Figura 4) presenta valores semejantes para suelos de bosques naturales reportados por Kawaguchi et al. (1995); Deng y Tabatabai (1996) y García-Izquierdo et al. (2003). En los primeros $5 \mathrm{~cm}$ de suelo (Figura 4) se observa en todos los casos una mayor actividad. Estos resultados concuerdan con estudios realizados por Deng y Tabatabai (1996) relacionados con la distribución de esta enzima, la que encontraron que está concentrada en la superficie del suelo y que disminuye con la profundidad. Por otra parte, esta enzima está correlaciona

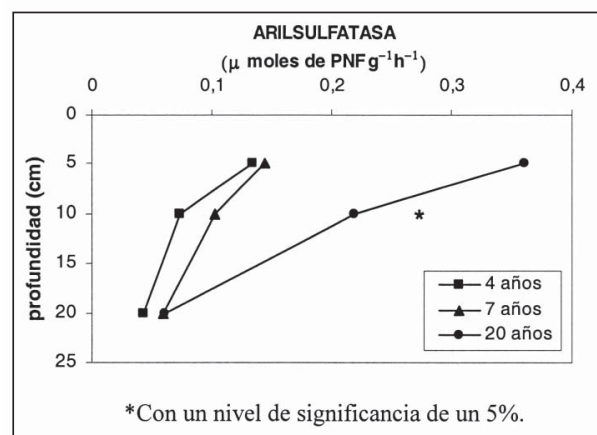

Figura 3: Actividad arilsulfatasa en un suelo Alfisol con diferentes años de CL.

Figure 3: Arilsulphatase activity at different depths in an Alfisol under NT from 4 to 20 years.

significativamente con el porcentaje de C orgánico (Cuadro 1), resultados que concuerdan con Eivazi y Tabatabai (1990), y Klose y Tabatabai (1999).

En Chile es primera vez que se determinan la actividad invertasa y CM-celulasa. Respecto a la actividad invertasa (Figura 4), no se observaron diferencias significativas entre 4 y 7 años de CL en los primeros 5 cm de suelo, pero sí en los de 5 a $10 \mathrm{~cm}$ y en los de 10 a $20 \mathrm{~cm}$ de profundidad. Con relación a la actividad CM-celulasa, los valores promedios para los primeros $5 \mathrm{~cm}$ de suelo, concuerdan con los reportados por Deng y Tabatabai (1996), quienes observaron que es mayor la actividad de ésta en suelos que son cultivados con CL que, en aquéllos que sufren diversas labores de arado.

Respecto a la actividad ureasa (Figura 5), los valores obtenidos son similares a los descritos por Trasar-Cepeda et al. (2000) y Alvear et al. (2005), y superiores a los reportados por Roldán et al. (2003). Con relación a las actividades enzimáticas proteasa-caseína y proteasa-BAA, al igual que la invertasa y CM-celulasa es primera vez que se determinan en Chile. Los valores encontrados para la proteasa-caseína y para la proteasa-BAA (Figura 5), son similares a los encontrados en suelos no intervenidos (Trasar-Cepeda et al., 2000). Por su parte, Klein y Koths (1980) encuentran 


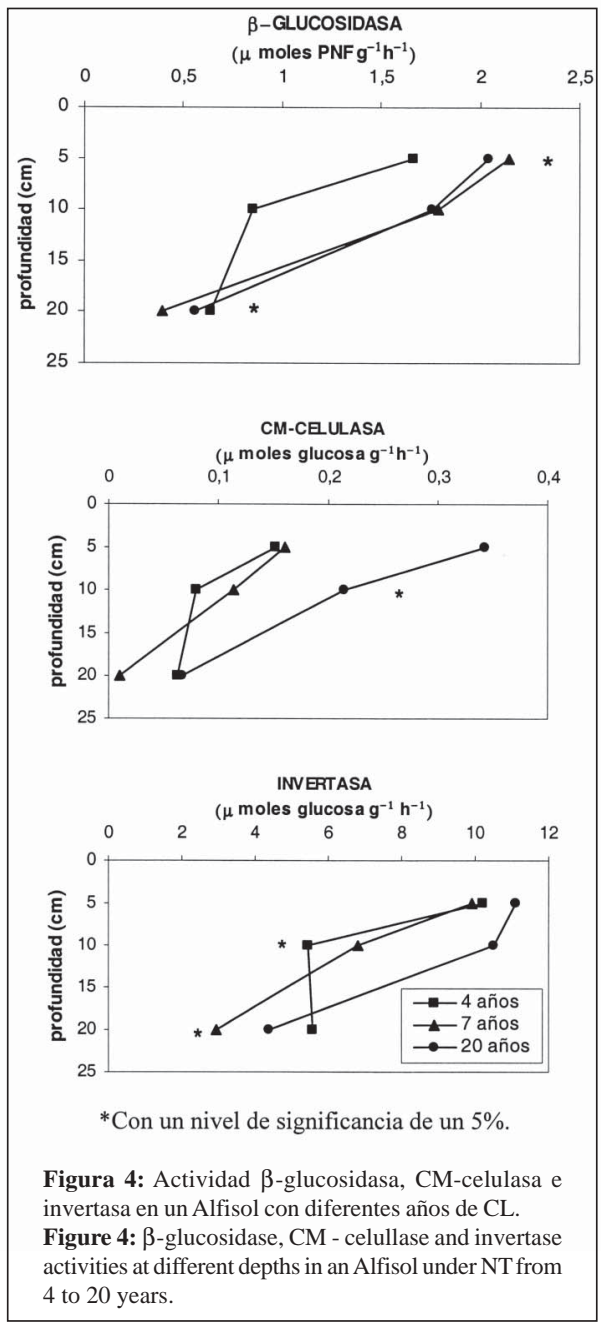

que la actividad proteasa caseína es mayor cuando el suelo es sometido a CL y Kandeler y Eder (1993) indican que la actividad proteasa caseína se incrementa cuando los suelos agrícolas se transforman en praderas. La actividad de la proteasa-BAA fue muy superior a la de las proteasas capaces de hidrolizar caseína en las tres profundidades ensayadas.

En general, se observa una gran correlación $(r>0.8 ; p<0.05)$ entre las diferentes actividades biológicas evaluadas (Cuadro 4), lo que concuerda con los resultados recientemente reportados por Alvear et al. (2005).

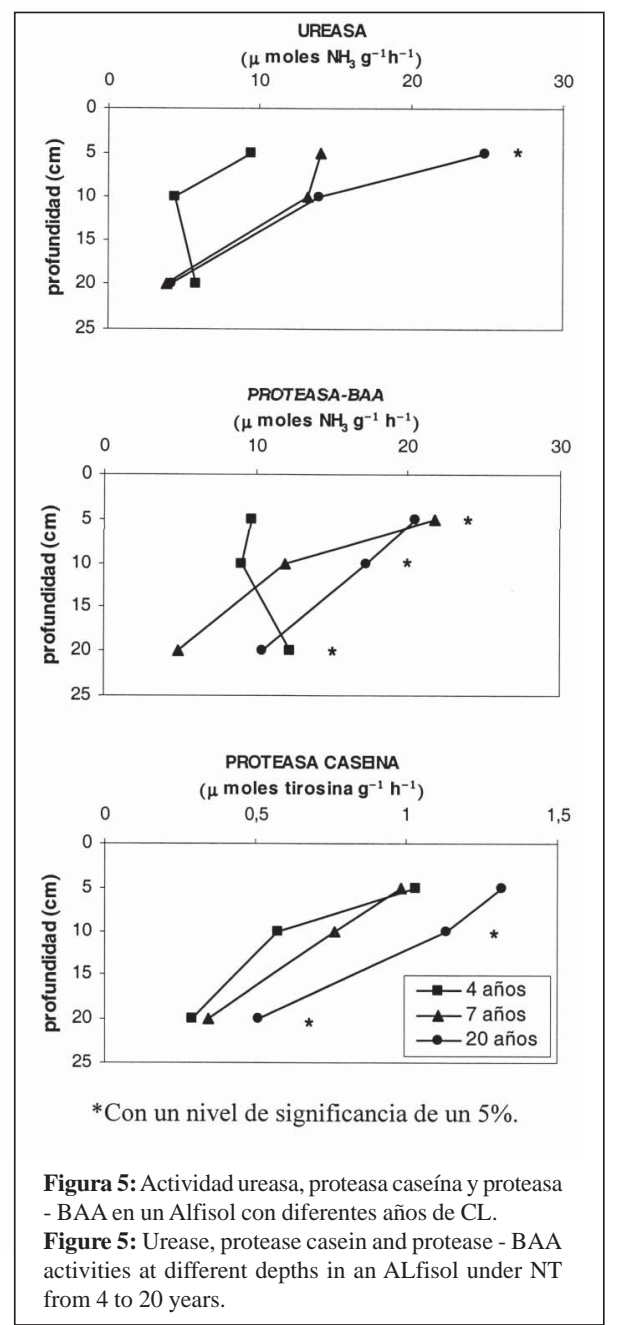

\section{CONCLUSIONES}

La CL en un Alfisol del sur de Chile, incrementó los niveles de MO a mayor número de años sometido a ésta práctica, y además aumentaron las actividades enzimáticas evaluadas, tanto del ciclo del C, P, S y N, así como también las actividades dehidrogenasa y catalasa, principalmente en los primeros $5 \mathrm{~cm}$ de profundidad del suelo.

La CL incrementó el C orgánico en los horizontes superficiales, disminuyendo las emisiones de $\mathrm{CO}_{2}$ a la atmósfera, mitigando en cierta medida, el efecto invernadero. 
El CBM, NBM, junto a la determinación de algunas actividades enzimáticas son sensibles a los cambios producidos en el entorno por efecto de los años de CL, la rotación y acumulación de residuos orgánicos.

Se observaron altos valores de correlación entre las variables estudiadas $(\mathrm{r}>0.8 ; \mathrm{p}<0.05)$, en la mayoría de las actividades biológicas evaluadas.

Los resultados encontrados, indican la importancia de las actividades biológicas para conocer acerca de lo que está ocurriendo en los agroecosistemas cuando éstos son manejados con CL, y el efecto de los diferentes tipos de rastrojos utilizados como cobertura vegetal. Sin embargo, estudios posteriores deberían estar orientados a analizar un mayor número de suelos chilenos, de diferente naturaleza edáfica, sometidos a labranza convencional y CL, para su comparación, de modo de extraer conclusiones más certeras acerca de la importancia que poseen las actividades biológicas en la evaluación temprana de los cambios producidos en los diferentes agroecosistemas y, por ende, en su sostenibilidad.

\section{AGRADECIMIENTOS}

Este estudio fue financiado por la Dirección de Investigación de la Universidad de La Frontera, Proyecto DIDUFRO No 9810. Los autores desean agradecer al Sr. Carlos C. Crovetto Lamarca por su disposición en la obtención de las muestras de suelo bajo cero labranza. Además, de agradecer al Convenio de Intercambio Universidad de La Frontera, Chile - Universidad Santiago de Compostela, España, que permitió que la Dra. M. Alvear hiciera una pasantía en los Laboratorios del Departamento de Edafología y Química Agrícola de la Universidad de Santiago de Compostela y en Laboratorios del Departamento de Bioquímica del Suelo, Instituto de Investigaciones Agrobiológicas de Galicia, CSIC, España.

\section{BIBLIOGRAFIA}

ACOSTA-MARTÍNEZ, V; CRUZ, L; SOTOMAYOR-RAMÍREZ, D and PÉREZ-ALEGRÍA， L. 2006. Enzymes activities as affected by soil properties and land use in a tropical watershed. Applied Soil Ecology, article in press.

ACOSTA - MARTINEZ, V. and TABATABAI, M. A. 2000. Enzyme activities in a limed agricultural. Soil Biology and Fertility of Soils 31: 85-89.

ACEVEDO, E. y SILVA, P. 2003. Agronomía de la Cero Labranza. Santiago, Universidad de Chile. Facultad de Ciencias Agronómicas No 10.132pp.

ALVEAR, M., ROSAS, A. ROUANET, J.L. y BORIE F., 2005. Effects of three soil tillage systems on some biological activities in an Ultisol from southern Chile. Soil and Tillage Research 82, 195-202.

ALVEAR, M., LOPEZ, R.,ROSAS, A.y ESPINOZA, N. 2006. Efecto de la aplicación de herbicidas en condiciones de campo sobre algunas actividades biológicas. Revista de la Ciencia del Suelo y Nutrición Vegetal 6, 64-76.

BADALUCCO, L. GELSOMINO, A., DELL ORCO, S. GREGO, S. and NANNIPIERI, P. 1992.Biochemical characterization of soil organic compounds extracted by $0.5 \mathrm{M}$ $\mathrm{K}_{2} \mathrm{SO}_{4}$ before and after chloroform fumigation. Soil Biology \& Biochemistry 24, (6):569-578.

BORIE, F., 1994. Microorganismos y cero labranza. Frontera Agrícola $\left(\mathrm{N}^{\mathrm{o}} 1\right) \mathrm{p}$ 13-26.

BORIE, F., RUBIO, R., MORALES, A. y CASTILLO, C., 2000. Relación entre densidad de hifas de hongos micorrizógenos arbusculares y pro- 
ducción de glomalina con las características físicas y químicas de suelos bajo cero labranza. Revista Chilena de Historia Natural 73:749-756.

BOWMAN, M. G. and TABATABAI, M. A., 1978. Phosphodiesterase activity of soils. Soil Science Society of American Journal 42:284-290.

BROOKES, P. C., LANDMAN, A., PRUDEN, G. and JENKINSON, D. S., 1985. Chloroform fumigation and the release of soil nitrogen: a rapid direct extraction method to measure microbial biomass nitrogen in soil. Soil Biology \& Biochemistry. 17:837-842.

CERÓN, L. E. y MELGAREJO, L. M., 2005. Enzimas del suelo: Indicadores de Salud y Calidad. Acta Biológica Colombiana 10, 5-18.

CROVETTO, C., 1992. Rastrojos sobre el suelo. Una introducción a la cero la branza. Editorial Universitaria. San tiago, Chile.312pp.

CROVETTO, C., 1996. Stubble over the soil. American Society of Agronomy. Madison,WI, 264pp.

CROVETTO, C., 2002. Cero Labranza: Los rastrojos, la nutrición del suelo y su relación con la fertilidad de las plantas.Trama Impresores S.A., Talcahuano, Chile. 225pp

DENG, S., and TABATABAI, M., 1996. Effects of tillage and residues management on enzyme activity in soil. I. Amidohidrolases. Biology and Fertility of Soils 22:202- 207.

DICK, R. P., RASMUSSEN, P. E. and KERLE, E. A., 1988 Influence of long-term residue management on soil enzyme activities in relation to soil chemical properties of a wheatfallow system. Biology \& Fertility of Soils 6:159-164.
DORAN, J. W., 1980. Soil microbial and biochemical changes with reduced tillage. Soil Science Society American Journal 44:765-771.

EIVAZI, F. and TABATABAI, M. A., 1990. Factors affecting glucosidase and galactosidase activities in soils. Soil Biology and Biochemistry 22:891-897.

FRANZLUEBBERS, A., 2002. Soil organic matter stratification ratio as an indicator of soil quality. Soil and Tillage Research 66, 95-106.

FRENEY, J R., 1961. Some observations on the nature of organic sulphur compounds in soil.Australian Journal of Agriculture Research 12:424-432.

GARCIA-ALVAREZ, A. and IBÁÑEZ, J., 1994. Seasonal fluctuations and crop. Influence on microbiota and enzyme activity in fully developed soils of Central Spain. Arid Soil Research and Rehabilitation 8:161-178.

GARCIA-IZQUIERDO, C., GIL-SOTRES, F., HERNÁNDEZ-FERNÁNDEZ,

T. y TRASAR-CEPEDA, C. 2003. Técnicas de Análisis Bioquímicos en suelos: Medida de Actividades Enzimáticas y Biomasa Microbiana. GGHT Ediciones y Mundi-Prensa, España, $371 \mathrm{p}$.

GAWRONSKA, A., KULINSKA, D., LENART, S., and JASKOWSKA, H., 1992. The effect of the maize monoculture on the biological properties of soil and on the yields of plants. Polish Journal Soil Science 25:89-94.

GIL-SOTRES, F., TRASAR-CEPEDA, M., CIARDI, C., CECCANTTI, B., and LEIROS, M., 1992. Biochemical characterization of biological activity in very young mine mine soils. Biology and Fertility of Soils 13, 25-30 
GROFFMAN, P. M., HANSON, GC., KIVIAT, E., and STEVENS, G. 1996. Variation in microbial biomass and activity in four different wetland types. Soil Science Society of American Journal 60:622-629.

JENKINSON, D., and LADD, N., 1981. Microbial biomass in soil: measurement and turnover. En: Paul E A \& Ladd $N$ (eds). Soil Biochemistry: : 415-471. Marcel Dekker, N.Y., Estados Unidos.

JOERGENSEN, R., 1996. Quantification of the microbial biomass by determining ninhydrin-reactive $\mathrm{N}$. Soil Biology and Biochemistry 22: 1023-1028.

JOERGENSEN, R., and EMMERLING, C., 2006. Methods for evaluating human impact on soil microorganisms based on their activity, biomass, and diversity in agricultural soils. Journal Plant Nutrition Soil Science 169, 295-309

KANDELER, E., and EDER, G., 1993. Effect of cattle slurry in grassland on microbial biomass on activities of various enzymes. Biology and Fertility of Soils 16: 249-254.

KANDELER, E., TSCHERKO, D., and SPIEGEL, H., 1999. Long-term monitoring of microbial biomass, $\mathrm{N}$ mineralization and enzyme activities of a Chernozen under different Tillage management. Biology \& Fertility of Soils 28: 343-351.

KAWAGUCHI, S., PEYARA, S. M., and YAMADA, Y., 1995. Soil properties and enzyme activity along narrow topographic environments of Salva series soils in Bangladesh. Institut of Tropical Agriculture 18:71-79.

KEENEY, D. R., and BREMNER, J. M.,1966. Comparison and evaluation of laboratory methods of obtaining an index of soil nitrogen availability. Agronomical Journal 58: 498-503.
KLEIN, T. M., and KOTHS, J. S., 1980. Urease, protease and acid phosphatase in soil continuously cropped to corn by conventional or non-tillage methods. Soil Biology and Biochemistry, 12: 293-294.

KLOSE, S., and TABATABAI, M., 1999. Arylsulfatase activity of microbial biomass in soils. Soil Science Society of American Journal 63:569-574.

LADD, J. N., and BUTLER, J. H. A., 1972. Short-term assays of soil proteolytic enzyme activities using protein and dipeptide derivatives as substrates. Soil Biology and Biochemistry 4:19-30.

MARTENS, R., 1995. Current methods for measuring microbial biomass $\mathrm{C}$ in soil: potentials and limitations. Biology and Fertility of Soils 19:87-99.

MELILlO, J. M., ABER, J. D., and MURATORE, J.F., 1982.Nitrogen and lignin control of hardwood leaf litter decomposition dynamics. Ecology 63, 621-626.

MERA, M. and ROUANET, J. L. 2003. Contribución de las leguminosas de grano en rotación con cereales: una revisión. Ca8. 4. En: Acevedo, E. (Ed) Sustentabilidad en cultivos anuales: Cero labranza y manejo de rastrojos. Santiago, Universidad de Chile, Facultad de Ciencias Agronómicas. Serie Ciencias Agronómicas № 8, pp 135-156.

NANNIPIERI, P., LANDI, L., and BADALUCCO， J., 1995. La capacità metabólica e la Qualità del suolo.Agronomia 29, 312-316.

OJEDA, G., 1996. Determinación de actividades biológicas en suelos volcánicos por medio de mediciones enzimáticas y de ATP. Tesis Licenciado en Bioquímica, Universidad Austral de Chile.Valdivia, Chile. 72p. 
OLANDER, L.P. and VITOUSEK, P.M., 2000. Regulation of soil phosphatase and chitinase activity by $\mathrm{N}$ and $\mathrm{P}$ availability. Biogeochemistry 49: 175-190.

PERUCCI, P., SCARPONI, L. and BUSINELLI, M., 1984. Enzyme activities in a clay loam soil amended with various crop residues. Plant \& Soil 81: 345-351.

PULFORD, I. and TABATABAI, M., 1988. Effect of water logging on enzyme activities in soils. Soil Biology and Biochemistry 20: 215-219.

ROLDÁN, A; CARAVACA, F; HERNANDEZ, M.T and GARCÍA, C. 2003. No-tillage, crop residue addition on soil quality characteristics under maize in Patzcuaro watershed (Mexico). Soil ad Tillage Research 72:65-73.

SAFFIGNA, P., POWLSON, D., BROOKES, P. and THOMAS, G., 1989. Influence of sorghum residues and tillage on soil organic matter and soil microbial biomass in on Australian vertisol. Soil Biology and Biochemistry 21:759-765.

SALAM, A. K., DESVIA, Y., SUTANTO, E., SYAN, T., NUGROHO, S. G. and KIMURA, M., 1999 Activities of soil enzymes in different land-use systems in middle terrace areas of Lampung Province, South Sumatra, Indonesia. Soil Science and Plant Nutrition 45 (1): 89-99.

SAUERBECK, D.R., 2001. CO2 emissions and $\mathrm{C}$ sequestration by agricultureperspectives and limitations.Nutrient Cycling in Agroecosystems 60, 253266.

SCHINNER, F. and VON MERSI, W., 1990. Xylanase-, CM-cellulase- and invertase activity in soil: and improved method. Soil Biology \& Biochemistry 22:511-515.
SKUJINS, J., 1978. History of abiotic soil enzymes research. En: R G Burns (Ed) Soil Enzymes: 1-49 Academic Press, London.

SMITH, J. and PAUL, E., 1990. The significance of soil microbial biomass estimations. Soil Biology and Biochemistry 6:357-396.

SPARLING, G., BRANDENBURG, S. and ZHU, CH., 1994 Microbial C and N in revegetated wheat belt soils in western Australia: estimation in soil, humus and leaf-litter using the ninhydrin method. Soil Biology and Biochemistry 26: 1179-1184.

TABATABAI, M. A., 1982. Page A L, Miller R H \& Keeney D R (eds) Soil Enzymes. En: Methods of Soil Analysis. Chemical and Microbiological Properties Part 2: 903-947 2nd Ed. Agronomy 9, ASA-SSSA. Madison.

TABATABAI, M. A., 1994. Enzymes. En: Weawer R, Augle S, Bottomly P, Bezdicek D, Smith S, Tabatabai A, Wollum A (eds). Methods of soil analysis. Microbiological and biochemical properties :775-833 Part 2., N ${ }^{\circ} 5$. Soil Science Society of America, Madison. Pp.

TABATABAI M, A. and BREMNER, J. M., 1970 Arylsulfatase activity of soil. Soil Science Society of American Proceedings. 34:225-229.

TRASAR-CEPEDA, C., LEIROS, M. C. and GIL-SOTRES, F., 1998. Towards a biochemical quality index for soils: an expression relating several biological and biochemical properties.. Biology and Fertility of Soils 26: 100-106.

TRASAR-CEPEDA, C., CAMIÑA, F., LEIROS, M. C. and GIL-SOTRES, F., 1999. An improved method to measure catalase activity in soils. Soil Biology and Biochemistry 31:483-485. 
TRASAR-CEPEDA, C., LEIROS, M. and GIL-SOTRES, F., 2000. Biochemical properties of acid soils under climax vegetation (Atlantic oakwood) in an area of the European temperatedhumid zone (Galicia, NW Spain): specific parameters. Soil Biology and Biochemistry 32:747-755.

VANCE, E. D., BROOKES, P. C. and JENKINSON, D. S., 1987. An extraction method for measuring soil microbial C Soil Biology \& Biochemistry 19:159-164.

VIDAL, I., ETCHEVERS, J. y FISCHER, A., 1997. Biomasa microbiana en un suelo sometido a diferentes manejos de labranza y rotación. Agricultura Técnica (Chile). 57:272-281.

VIDAL, I. y TRONCOSO, H., 2003. Manejo de rastrojos en cultivos bajo cero labranza. Cap. 4. En: Acevedo, E. (Ed) Sustentabilidad en cultivos anuales: Cero labranza y manejo de rastrojos. Santiago, Universidad de Chile, Facultad de Ciencias Agronómicas. Serie Ciencias Agronómicas No 8, pp 57-81.

ZAGAL, E., RODRÍGUEZ, N., VIDAL, I. y QUEZADA, L. 2002. Actividad microbiana en un suelo volcánico bajo distinto manejo agronómico. Agricultura Técnica 62(2): 297-309. 